\title{
Design Research of Photovoltaic Inverter
}

\author{
Zhe Bei Wang ${ }^{1, a}$, Hong Hua Wang ${ }^{1, b}$, Cheng Liang Wang ${ }^{2}$,Lin Cui ${ }^{3}$ \\ ${ }^{1}$ College of Energy and Electrical Engineering, Hohai University, Nanjing, China ,211100 \\ ${ }^{2}$ Jiangsu Frontier Electric Technology Co., Ltd, Nanjing, China ,211102 \\ ${ }^{3}$ Jiangsu Electric Power Company Research Institute, Nanjing, China, 211103 \\ ª407507155@qq.com, bwanghonghua@263.net
}

Keywords: two-stage filter, inverter strategy of SVPWM, sine wave

\begin{abstract}
Based on the practical engineering application, This paper analyses the inverter strategy of SVPMW. Aiming at the refining problem of sine function value table, on the basis of unrefined sine function value table, through additional two-stage filter, the inverting result of the inverter is greatly improved. The harmonic wave is effectively suppressed and the output wave of the inverter is much more close to sine wave. At last, the Matlab simulation and setting up of the experimental platform of the inverter validate the effectiveness of the additional two-stage filter.
\end{abstract}

\section{Introduction}

Along with the shortage of traditional energy and growing demand of energy resource, the exploitation and application of new energy have become the inevitable trend in today's world development[1,2]. As one kind of clean energy, solar energy has gained more and more attention of people. However, PV panel can only convert solar energy to DC electric power, which can't meet AC load demands of daily production and life. By means of PV inverter which can convert DC electric power to common AC electric power, the utilization of solar energy technology has obtained terrific promotion.

Inverter technology is one of the basic forms of electric power conversion. In general, inversion means using power electronic devices and trigger strategy to convert DC to AC which is provided for the load or power grid. In recent years, space vector theory of motor is introduced to inverter and inverter's control. Increasingly, the thought of SVPWM control is formed and developed. The principle of the thought is utilizing different combination of bridge arm switch signal of the inverter to make the moving trajectory of the output voltage space vector of the inverter approach to a circle as close as possible[3]. SVPWM inverter strategy has been widely applied to different kinds of inverters because of the advantage of higher utilization of DC voltage, lower AC harmonic wave against other strategies.

\section{Design and improvement of the inverter}

The main circuit of PV inverter is showed as figure 1, which is composed of DC power supply, inverter bridge, filter circuit and three-phase load. In this paper, the traditional three-phase bridge inverter structure is adopted and MOSFET transistor is choosed as the switch transistor to construct the main circuit of the inverter. 


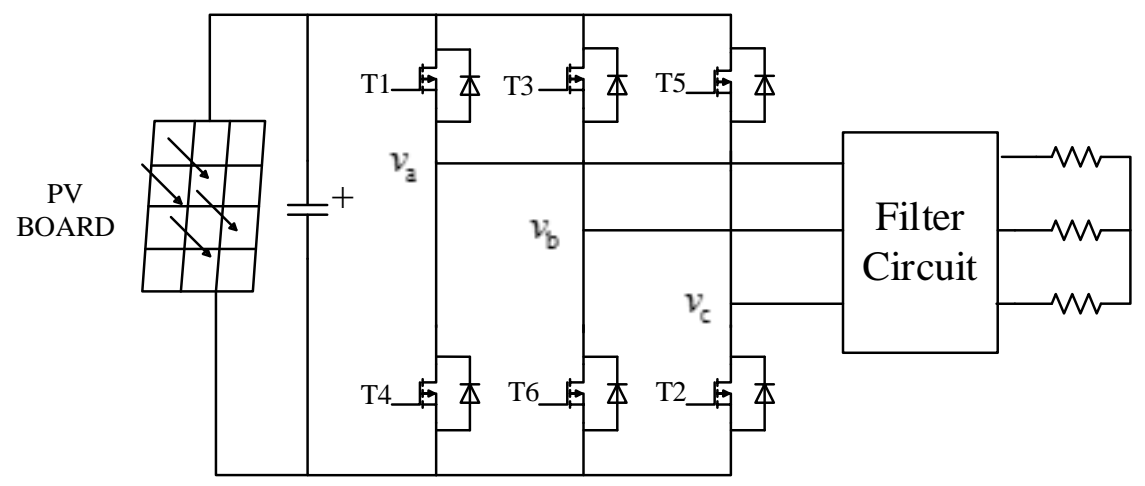

Fig.1 Structure of the inverter

Analysis of SVPWM inverter strategy. Usually, a space vector in a cycle can be equally divided into six sectors, each sector holds $60^{\circ}$. By linear combination of two basic neighbored voltage space vectors in each sector, we can get a rotating voltage space vector which has invariable amplitude and angle speed.

As showed in figure 2, $U_{x}$ and $U_{x \pm 60}$ represent two basic neighbored voltage vectors in any sector. $U_{\text {out }}$ is the reference phase-voltage vector, the amplitude is the amplitude value of phase-voltage, rotating angle speed is the angle speed of the output sine voltage. From figure 1, we can find that $U_{\text {out }}$ can be described as:

$$
U_{\text {out }}=\frac{t_{1}}{T_{P W M}} U_{x}+\frac{t_{2}}{T_{P W M}} U_{x \pm 60}
$$

In Eq. 1, $t_{1}$ and $^{t_{2}}$ respectively stands for the action time of $U_{x}$ and $U_{x \pm 60}$; $T_{P W M}$ is the action time of $U_{\text {out }}$. During the next $T_{P W M}$ period, $U_{x}$ and $U_{x \pm 60}$ are still employed to conduct linear combination. In order to make sure that the amplitude value of combined voltage space vector $U_{\text {out }}$ is consistent with the original value, the action time $t_{1}$ and ${ }^{t_{2}}$ should be changed. During each $T_{P W M}$ period, the action time of the basic neighbored vector is altered to guarantee the equality of the amplitude value of all combined voltage space vector. Thus, if $T_{P W M}$ is small enough, the trajectory of voltage space vector is an equilateral polygon whose shape approximates to a circle.

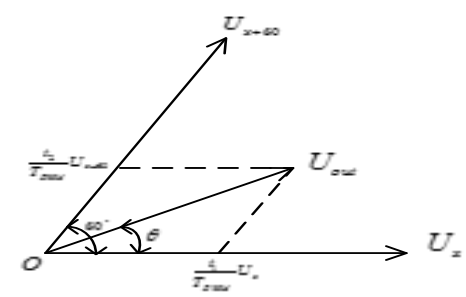

Fig.2 linear combination of voltage space vector 


$$
\left.\begin{array}{c}
t_{1}=\frac{2 U_{\text {out }}}{\sqrt{3} U_{x}} T_{P W M} \sin \left(60^{\circ}-\theta\right) \\
t_{2}=\frac{2 U_{\text {out }}}{\sqrt{3} U_{x \pm 60}} T_{P W M} \sin \theta
\end{array}\right\}
$$

In which, $T_{P W M}$ and $U_{\text {out }}$ is predetermined; $\theta$ can be determined by the product by output sine voltage angle speed $\omega$ and $n T_{P W M}$. Thus, when the two basic neighbored voltage space vector $U_{x}$ and $U_{x \pm 60}$ are determined, in Eq. 2, the action time of $t_{1}$ and $t_{2}$ are accordingly determined.

In order to modulate the angle speed $\omega$ to achieve the purpose of frequency conversion, zero vector action time $t_{0}$ is added during $T_{P W M}$ period, there is:

$$
T_{P W M}=t_{0}+t_{1}+t_{2}
$$

For purpose of making the speed of flux linkage smooth, zero vector is generally equally divided into several parts which will be inserted into the trajectory of the flux linkage in a multi-point way, however the sum of the action time is still $t_{0}[4,5]$.

At present, the popular seven section PWM wave of voltage space vector consists of three section zero vectors and four neighbored non-zero vectors. Besides, the three zero vectors respectively locate in the beginning, center, and end of the PWM wave[6,7]

Improvement of the inverter. According to Eq. 2, the calculation of $t_{1}$ and $t_{2}$ needs the help of sine function value table, which means the refining degree directly decides the achievable $T_{P W M}$ in the inverter. While $T_{P W M}$ is small enough, the output wave of the inverter approximates to sine wave. If the sine value table is refined, there is plenty of storage space required to save sine function value, which is adverse to the realization of the inverter. Conversely, if the table isn't refining enough, the inverted wave is comparatively rough. And the wave may have harmonic component even distortion. Correspondingly, the expected effect of inverter is unreachable. It is thus clear that the refining degree of the sine function value table is closely related to the output of the inverter. In the application of the inverter, however, the impact that the table's limited by the storage space of the controller should not be too massive.

This paper adds a two-stage RC filter circuit between the output end and the load, so as to reduce the harmonic component in the inverted output wave without amplifying the storage space of the sine function value table. Compared with the traditional inverter which adopts only one-stage filter circuit, the employment of two-stage filter circuit can remove the still larger harmonic component after the first stage filtering, which ensures the better output wave of the inverter. For the sake of highlighting the impact of rough sine function value table, there are 90 sine values stored in the 
table, the interval among the values is $1^{\circ}$.

The required frequency of sine wave is $50 \mathrm{~Hz}$, in terms of one-order LPF(Low-Pass Filtering) transfer function:

$$
H(\mathrm{~s})=\frac{1}{s C R+1}
$$

With the passband edge frequency substituted to Eq.4, there is

$$
\frac{1}{\sqrt{\left(f_{c} \mathrm{RC}\right)^{2}+1}}=\frac{1}{\sqrt{2}}
$$

In which, $f_{c}$ is the passband edge frequency.

In this paper, passband edge frequency of the first-stage filter is $100 \mathrm{~Hz}$, passband edge frequency of the second stage is $60 \mathrm{~Hz}$. With simple calculation, the required parameters are: $R_{1}=10 \mathrm{~K} \Omega, C_{1}=1 \mu \mathrm{F}, R_{2}=1.7 \mathrm{~K} \Omega, C_{2}=10 \mu \mathrm{F}$. The structure of the two-stage RC filter is showed in the figure below.

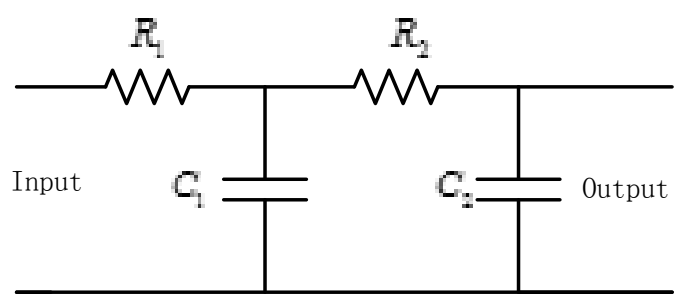

Fig.3 Structure of the two-stage RC filter

\section{Simulation Experiment}

The simulation model of the inverter is set up with MATLAB/Simulink and showed in figure 4 . Universal bridge/Mosfet is choosed to compose the three-phase bridge inverter; 7 section voltage space vector PWM wave is generated by Discrete PWM Generator whose carrier frequency is 18 $\mathrm{KHz}$. The three-phase load is a $10 \mathrm{~K} \Omega$ resistor.

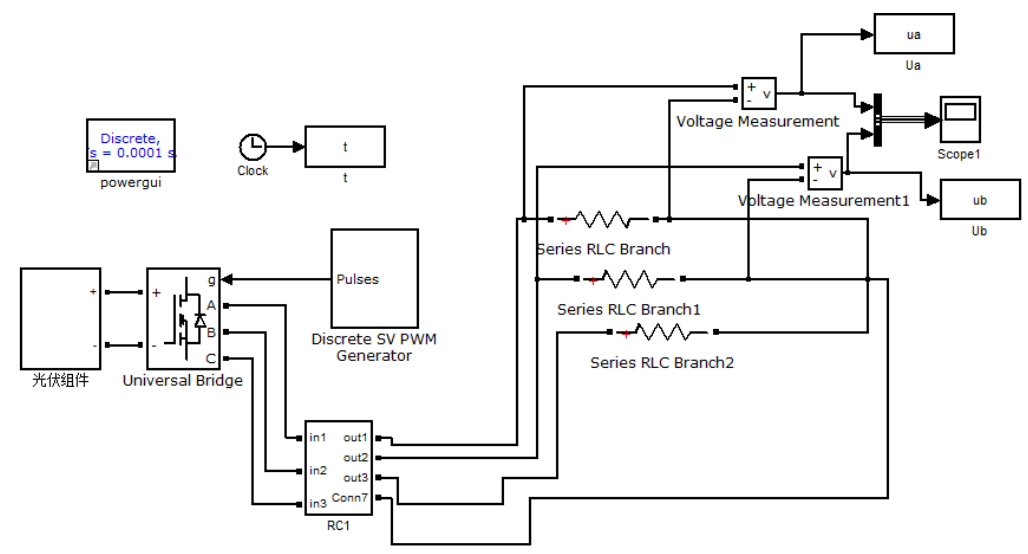

Fig.4 Structure of inverter simulation

If there is only one-stage RC filter, the phase voltage of the inverter output $A$ and $B$ is showed in Fig.5. We can easily find that the distortion of the phase voltage, the inverting effect is not satisfying. 


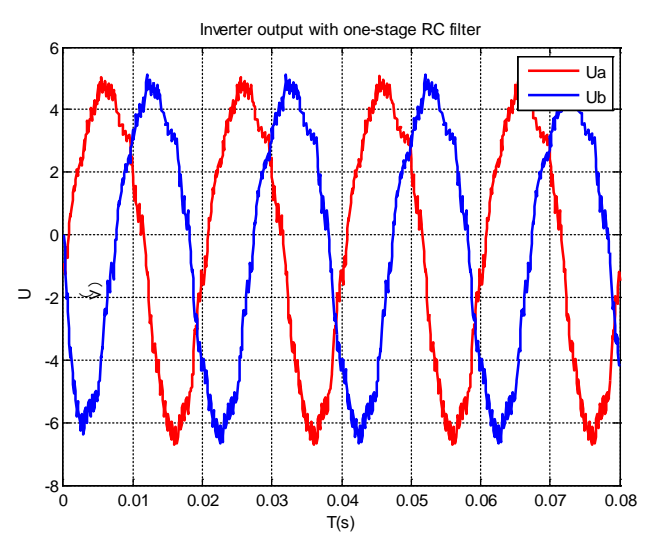

Fig.5 Inverter output with one-stage RC filter

If we adopt two-stage RC filter, the phase voltage of the inverter output $A$ and $B$ is showed in Fig.6. The output of the inverter phase voltage is close to sine wave, the inverting effect is ideal.

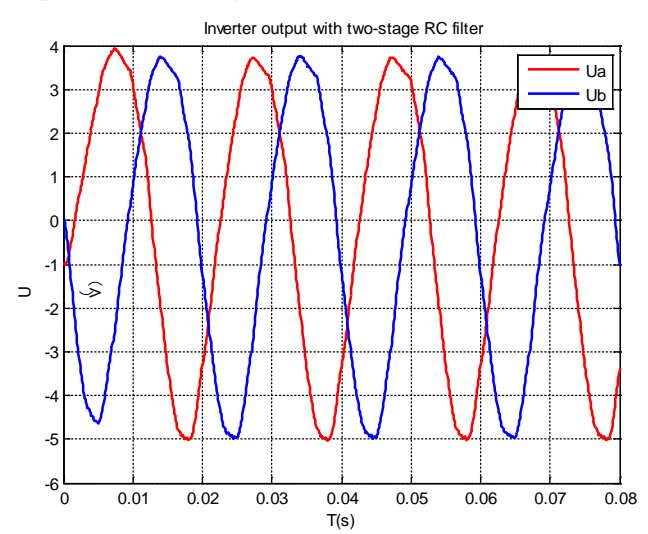

Fig.6 Inverter output with two-stage RC filter

\section{Experimental Verification}

In this paper, TMS320F2812 chip is selected to realize the control strategy of SVPWM. This chip has a high dominant frequency of $150 \mathrm{MHz}$. The internal hardware circuit of the chip has two functional event manager. Each manager can generate 3 pairs of complementary PWM waves which has dead time. Base on the properties given above, the design of the hardware and software of the inverter is greatly simplified, meanwhile, the accuracy and stability of the inverter is hugely improved[8,9,10].

There is an opto-coupler between the output signal of TMS320F2812 and main circuit of the inverter. The opto-coupler can effectively protect the control circuit. In this paper, we choose HCPL0631 as the fast opto-coupler. Because the main circuit of the inverter is three-phase bridge inverter circuit, so the drive chip could be IR2136 which is qualified for high frequency work. The dead time of IR2136 is 250ns and on/off time is 400ns. Apart from this, IR2136 has the ability of dead zone protection, which means the chip can effectively protect the main circuit. As for MOSFET, we choose IRF3205, which is highly reliable and solid. We choose a $10 \mathrm{~K} \Omega$ resistor as the analog three-phase load which is star join. From the above mentioned, the two-stage filter SVPWM inverter experimental platform is constituted, which is showed in Fig.7. 


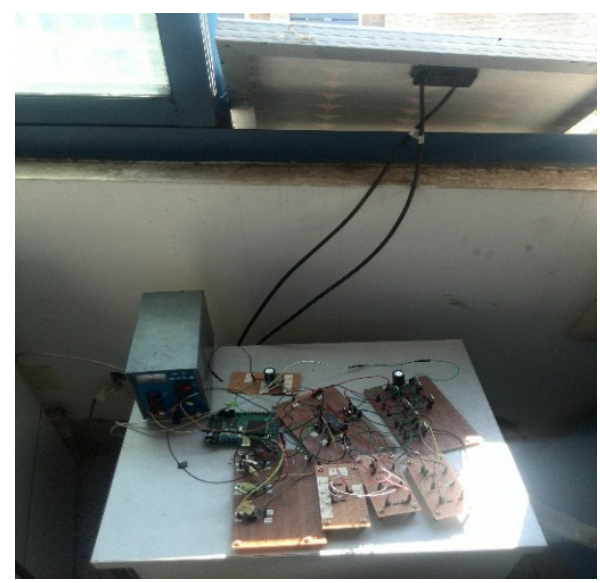

Fig.7 Experimental platform of PV inverter

The real output of the SVPWM inverter with one-stage inverter is showed in Fig.8. According to the phase voltage wave of phase A and B of the inverter filtered by one-stage RC filter, we can find that the filtering effect of the one-stage RC filter is not ideal. In terms of Fig. 9, by conducting the FFT analysis of wave of phase A, it is easily found that the one-stage RC filter has some inhibiting effect on the harmonic effect of the output wave, however, the effect isn't obvious enough. The second and higher harmonic wave still exists, the inverting output wave has not reached the expected inverting effect.

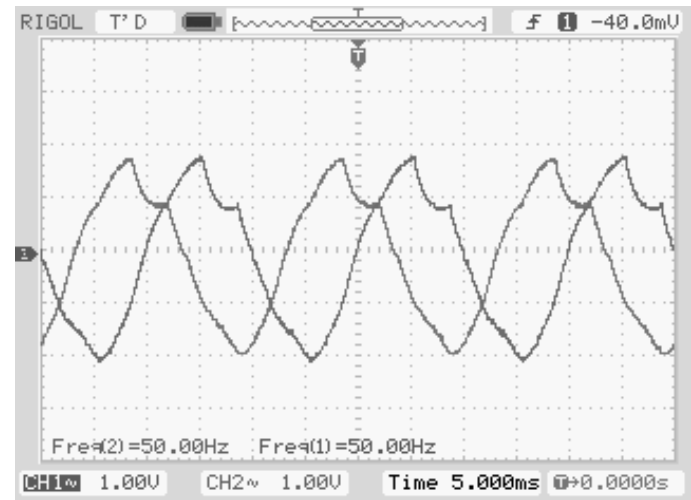

Fig.8 Wave of phase A and B of SVPWM inverter with one-stage filter

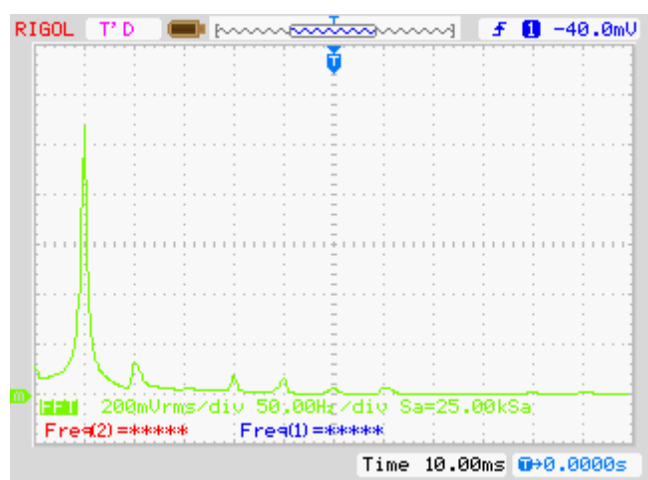

Fig.9 FFT analysis of A phase wave of SVPWM inverter with one-stage filter

In order to improve the distortion of the output wave of SVPWM inverter with one-stage filter, we design SVPWM inverter with two-stage filter, the wave of phase A and B is showed in Fig.9, from which, we can find that the phase difference is $120^{\circ}$ and the wave approximates to sine wave without any distortion. Clearly, the two-stage RC filter effectively improve the inverting effect. In terms of Fig. 9, by FFT analysis of wave of phase A, the second and higher harmonic wave is almost filtered, and the output wave is vastly modified so as to achieve the expected outcome. 


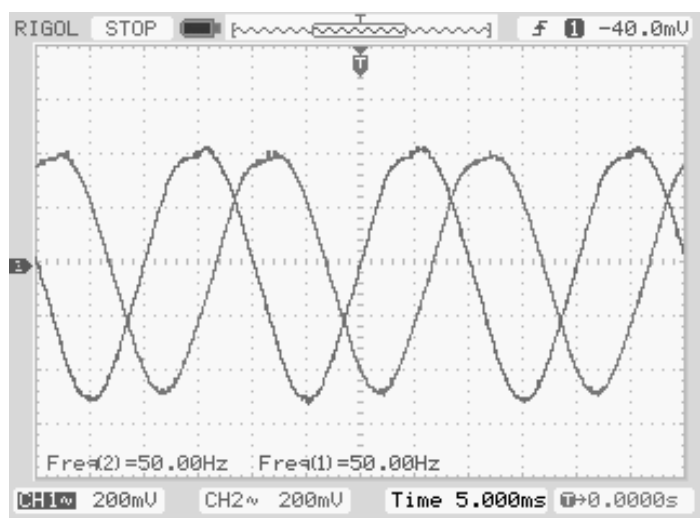

Fig.10 Wave of phase A and B of SVPWM inverter with two-stage filter

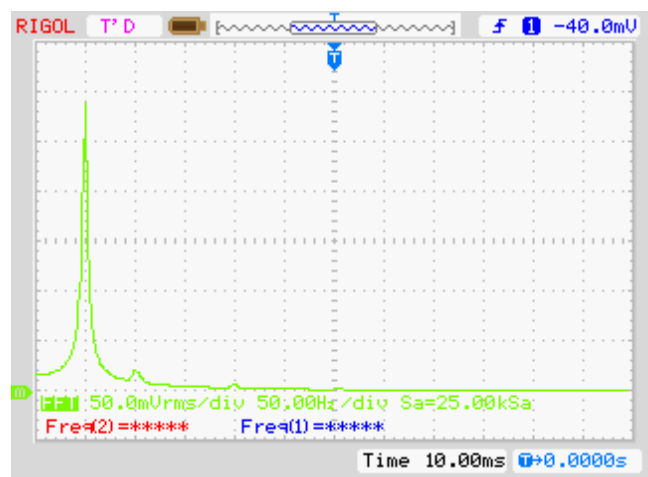

Fig.11 FFT analysis of A phase wave of SVPWM inverter with one-stage filter

\section{Conclusion}

This paper introduces the basic theory of SVPWM inverter modulation strategy and analyzes the deficiency of this strategy in the practical application. Based on the analysis above, the two-stage filtering method is proposed to improve the deficiency. By adopting two-stage RC filtering between the inverter and load, we can make the refining sine function value table needn't take plenty of place in the control chip. By experiment and simulation, the effectiveness of two-stage RC filter. According to the facts that there is no apparent distortion in the output wave through two-stage RC filtering and the wave is close to sine wave, the prospect and range of application of inverter is enlarged.

\section{Acknowledgements}

This research was supported by a grant from the State Grid Jiangsu electric power company project of China (No.J2014028).

\section{References}

[1] Luo Ming, Yang Jingming. The research of two-stage photovoltaic maximum power point tracking system[J]. Power Electronics,2009(5):20-21.

[2] Wu Guo, Wu Sijiang, Wang Wei. The technology of SVPWM and its application in photovoltaic power generation systems[J]. Science and technology and enterprise,2014(9):340-342.

[3] Xu Hui, Xiao Lan, Yan Yangguang. The research of digital voltage regulation with SVPWM in inverter[J]. Journalof Nanjing University of Aeronautics \& Astronautics,2002,34(5):456-460. 
[4] Wang Xiaoming. The DSP control of motors- TI company's DSP applications(The second edition)[M]. Beihang University Press.

[5] Altas I H, Sharaf A M. A novel on-line MPPT search algorithm for PC arrys[J].Energy Conversion,IEEE Transactions on,1996,11(4):748-754.

[6] Femia N ,Petrone G, Spagnuo G,et al. Optimization of perturb and observe maxium power point tracking method[J]. IEEE Transactions on Power Electronics,2005,20(4):963-973.

[7] Gyugyi L,Strycula E C.Active ac power filters[C].In:Proc of IEEE/IAS Annual Meeting,1976:529-535.

[8] Akagi H, Kanazawa Y, Nabae A.Generalized theory of the instantaneous teactive power in three-phase circuits[C].In:IEEE\&JIEE.Proceedings IPEC.Tokyo:IEEE,1983:1375-1386.

[9] Infield D G,Onions P,Simmons A,et al.Power quality from multiple grid-connected single-phase inverters[J].Power Delivery,IEEE Transactions on,2004,19(4):1983-1989.

[10]Bo cao,Liuchen chang,Li H.Implementation of the RBF neual network on a SOPC for maximum power point tracking[J].Electrical and Computer Engineering,2008:981-986. 